\title{
Fibroblast radiosensitivity and intraocular fibrovascular proliferation following radiotherapy for bilateral retinoblastoma
}

\author{
DANIEL M ALBERT, ${ }^{2}$ DAVIDS WALTON,${ }^{2}$ RALPH R WEICHSELBAUM, ${ }^{2}$ \\ J ROBERT CASSADY, ${ }^{2}$ JOHN B LITTLE, ${ }^{3}$ DONNA LEOMBRUNO ${ }^{4}$ \\ RAMANA TRANTRAVAHI ${ }^{4}$ AND CARMEN A PULIAFITO'
}

From the 'Department of Ophthalmology, Harvard Medical School, Massachusetts Eye and Ear Infirmary; ${ }^{2}$ Joint Center for Radiation Therapy, Department of Radiation Therapy, Harvard Medical School; ${ }^{3}$ Department of Cancer Biology, Harvard School of Public Health; and the ${ }^{4}$ Cytogenetics Laboratory, Dana Farber Cancer Institite, Boston, MA and Department of Medicine, Harvard Medical School, USA.

SUMmARY A 5-day-old female patient was found to have large hereditary retinoblastomas in the posterior pole of each eye. The patient received radiation treatment over a 39-day period, with each retina receiving $4600 \mathrm{rad}$. Two weeks after the complete treatment the tumours had regressed to approximately one-quarter of their original size. By 14 weeks following completion of radiotherapy the patient had developed in each eye extensive iris neovascularisation with progressive closure of the filtration angles, secondary glaucoma, and retinal detachments resulting from fibrovascular proliferation on the retinal surface. Radiosensitivity studies were from separate conjunctival biopsies obtained before and after radiation. These showed a $\mathrm{D}_{0}$ (calculated survival curve parameters, defined in the Methods section) in the exponential growth phase of 110 prior to radiation and a postirradiation exponential growth phase $\mathrm{D}_{0}$ of 70 . Karotype studies showed several chromosomal abnormalities following radiotherapy. The clinical course and pathology findings are thought to represent an unusually severe orbital and ocular response to radiation therapy. These findings are consistent with our hypothesis that some patients with hereditary retinoblastoma may have a defect in the accumulation repair of $x$-irradiation induced DNA damage.

\begin{abstract}
Recent studies have shown that fibroblast cultures from patients with hereditary retinoblastoma have an increased mean radiosensitivity when compared with fibroblasts obtained from normal controls or fibroblasts from the non-hereditary group. ${ }^{12}$ This observation has been of particular interest because patients with hereditary retinoblastoma, as generally determined by bilateral involvement or a known family history of the disease, subsequently develop second tumours at sites distant from the orbit as well as within the irradiated field following radiotherapy. ${ }^{3}$ We recently studied fibroblast sensitivity to $x$-irradiation in an infant with bilateral retinoblastoma before and after radiation therapy. Increased sensitivity to

Correspondence to Daniel M Albert, MD, Massachusetts Eye and Ear Infirmary, 243 Charles Street, Boston, Massachusetts, 02114, USA.
\end{abstract}

$x$-irradiation and multiple chromosomal abnormalities in conjunctival fibroblast cultures were observed following treatment. Moreover, this patient had an unusually severe clinical response to radiotherapy, with a rapid onset of iris neovascularisation and preretinal fibrovascular proliferation which led to loss of both eyes despite regression of the intraocular tumours.

\section{Case report}

The patient, a girl, was found to have large retinoblastomas in the posterior pole of each eye at the age of 5 days. The patient's father had a history of bilateral retinoblastoma which had been treated by enucleation of one eye and radon seed implantation in the other. At the time of initial diagnosis there was 
no evidence of intraocular neovascularisation. Bone marrow aspiration and lumbar puncture showed no evidence of tumour cells.

Radiation therapy of both eyes was carried out for a total of 23 treatments over 39 days. A $4 \mathrm{MeV}$ linear accelerator was used, with the patient under anaesthesia with ketamine hydrochloride. Both eyes were treated by anterior and lateral portals with a lens block used on the right eye for the full treatment and on the left eye for the last six days. Initially, $600 \mathrm{rad}$ were given to the lateral fields without anterior supplement. Then the full four fields received the remainder to a total dose of $4600 \mathrm{rad}$. It was estimated that the right iris with the lens block received a minimum dose of $690 \mathrm{rad}$ to a maximum dose of $2230 \mathrm{rad}$ at the periphery. The retina in that eye received $4600 \mathrm{rad}$ except for a small portion directly posterior to the lens. The left iris received a minimum of 1754 rad to a maximum of $2230 \mathrm{rad}$, and the retina again received $4600 \mathrm{rad}$.

Substantial tumour regression was noted soon after radiotherapy. Two weeks after the completion of the treatment course the tumours had regressed to one-quarter of their original size. The patient was examined under anaesthesia at monthly intervals. When examined at age 5 months- 14 weeks following completion of radiotherapy-she was found to have extensive iris neovascularisation, partial closure of the filtration angle in each eye, and associated secondary glaucoma. Retinal detachments in each eye were found. No new tumour or recurrence of tumour growth occurred. Because of intractable neovascular glaucoma and pain no evidence of visual function in either eye, enucleation of the left and then the right eye was performed. The patient progressed satisfactorily after the operation without evidence of orbital or distant tumour. Striking shrinkage of the orbital space has occurred, associated with dry conjunctiva sockets. Her gross motor development has been slow.

\section{Materials and methods}

\section{RADIOSENSITIVITY STUDIES}

Small conjunctival biopsy specimens were obtained at the time of initial examination under anaesthesia and diagnosis (designated B-0821), and at the time of enucleation (following radiotherapy) (designated B814). Fibroblast cultures were established by methods previously described. ${ }^{4}$

For survival experiments exponentially growing cells were detached with $0.25 \%$ trypsin in calciumfree and magnesium-free Earle's balanced salt solution and seeded at appropriate numbers into triplicate $10 \mathrm{~cm}$ diameter Falcon tissue culture dishes containing $10 \mathrm{ml}$ of culture medium. Feeder layers were not used, and the density of cells in these experiments never exceeded $4 \times 10^{4} /$ dish. Dishes were irradiated 18 . hours later (multiplicity $=1$ ) at room temperature and ambient atmosphere with a $220 \mathrm{KVp}$ General Electric Maximar unit operated at $15 \mathrm{ma}$ and yielding a dose rate of $80 \mathrm{rad} / \mathrm{minute}$ as determined by a Victoreen ionisation chamber. The half-value layer of the beam was $0.5 \mathrm{~mm}$ of copper. At least four doses, ranging from 50 to $700 \mathrm{rad}$, were used per experiment. After irradiation the dishes were returned to the incubator, the medium was changed every five to seven days, and the colonies were fixed and stained after 12 to 21 days. Satellite colony formation was not observed under these conditions. Experiments were scored under a dissecting microscope with colonies composed of 50 or more

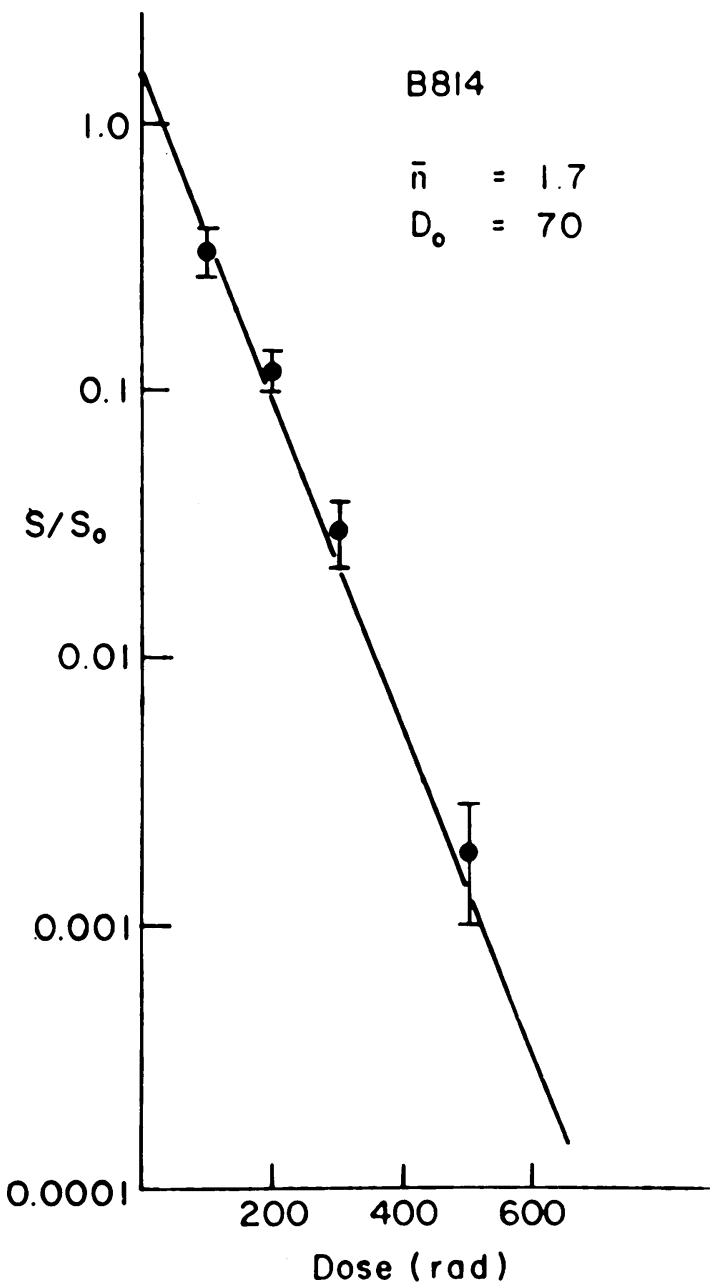

Fig. 1 Graph shows x-ray survival curve parameters determined by least-squares regression analysis for cultured fibroblasts from preirradiation period. $D_{0}=110, n=1.6$. 
cells considered as survivors. Cloning efficiencies ranged from 4.0 to $97.5 \%$. The calculated survival curve parameters were the $D_{0}$ in rad (inverse of the slope of the straight-line portion of the survival curve) and $n$ (a measure of the magnitude of the shoulder region of the survival curve). They were both derived from a least-squares regression analysis of points above $100 \mathrm{rad}$.

To eliminate the effects of differences in cell cycle distribution accounting for differences in radiosensitivity in cultures derived from pre- and postradiotherapy biopsies, $x$-ray survival experiments were performed on density-inhibited plateau phase cultures. Over $90 \%$ of cells in these cultures are in the $\mathrm{G}_{1}$ phase of the cell cycle. Cultures were grown to confluence and the medium changed daily for three days. Irradiation was done on the fourth day. Cells were subcultured immediately after irradiation.

\section{CHROMOSOME STUDIES}

Logarithmically growing cultures of both the cell lines B-8021 (the preradiation cell line) and B-814 (the postirradiation cell line) were treated with demecolcine (Colcemid) at a final concentration of $0 \cdot 1 \mu \mathrm{g} / \mathrm{ml}$ and slides were stained with quinacrine mustard $(50 \mu \mathrm{g} / \mathrm{ml})$ as described previously. ${ }^{5}$ Well spread and well banded metaphases were photographed with a Leitz Ortholux microscope equipped with an Orthomat camera. The prints were made on Agfa paper.

\section{PATHOLOGY}

The eyes were obtained at the time of enucleation and immediately placed in glutaraldehyde-formalin fixative. They were embedded in paraffin, sectioned, and examined by haematoxylin and eosin and periodic acid Schiff stains in accordance with methods previously decribed.

\section{Results}

Figs. 1 and 2 show $x$-ray survival experiments from separate biopsies obtained before and after treatment with $4600 \mathrm{rad}$ in 39 days. The $\mathrm{D}_{0}$ of the preradiotherapy biopsy of cells in the exponential growth phase was $110(n=1 \cdot 6)$; in the plateau phase $D_{0}$ was $111(n=22)$ (immediate subculture; data not shown). The $\mathrm{D}_{0}$ of the postirradiation biopsy in the exponential phase of growth is $70(n=1 \cdot 8)$ (Fig. 1) and the plateau culture phase $\mathrm{D}_{0}=85$, after immediate subculture (data not shown).

\section{HISTOPATHOLOGY}

The histological appearance of both eyes was similar. The residual tumours were limited to several small foci with marked calcification and necrosis (Fig. 3a).
No cytologically viable tumour cells were seen. Marked iris neovascularisation with closure of the filtration angle was noted (Fig. 3b). Extensive preretinal fibrovascular membranes were present, with large traction retinal detachments observed. The retina itself showed extensive loss of ganglion cells, as well as marked cystic changes in the inner retinal layers (Fig. 3c). Occasional retinal vessels were seen to be narrowed and obliterated.

\section{CHROMOSOME STUDIES}

Fourteen metaphases were photographed and analysed, and four of these were karyotyped from a fibroblast culture obtained prior to radiotherapy (B-8021) (Fig. 4). All cells showed a diploid female karyotype. Five of the 14 metaphases showed random chromosome loss due to cell breakage. The fibroblast cell line obtained after radiation therapy (B-814) also had a modal number of 46 chromosomes (Fig. 5). In seven

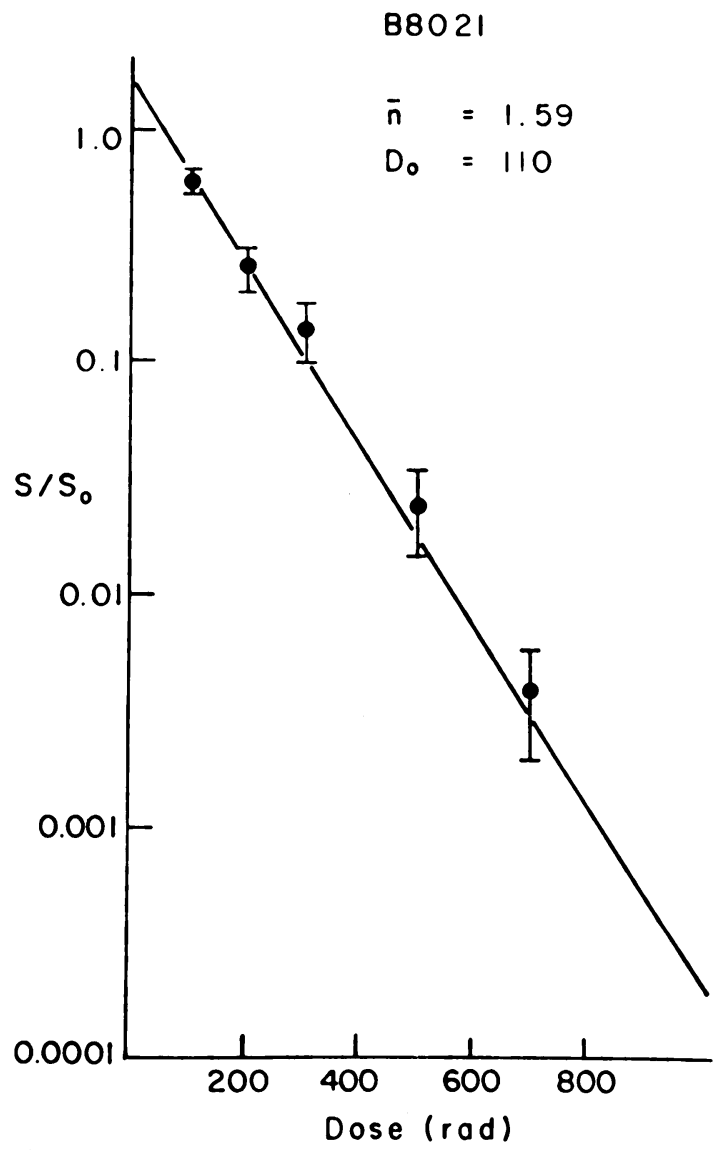

Fig. 2 Graph shows x-ray survival curve parameters determined by least-squares regression analysis following radiotherapy. Postirradiation $D_{o}=70, n=1 \cdot 7$. 


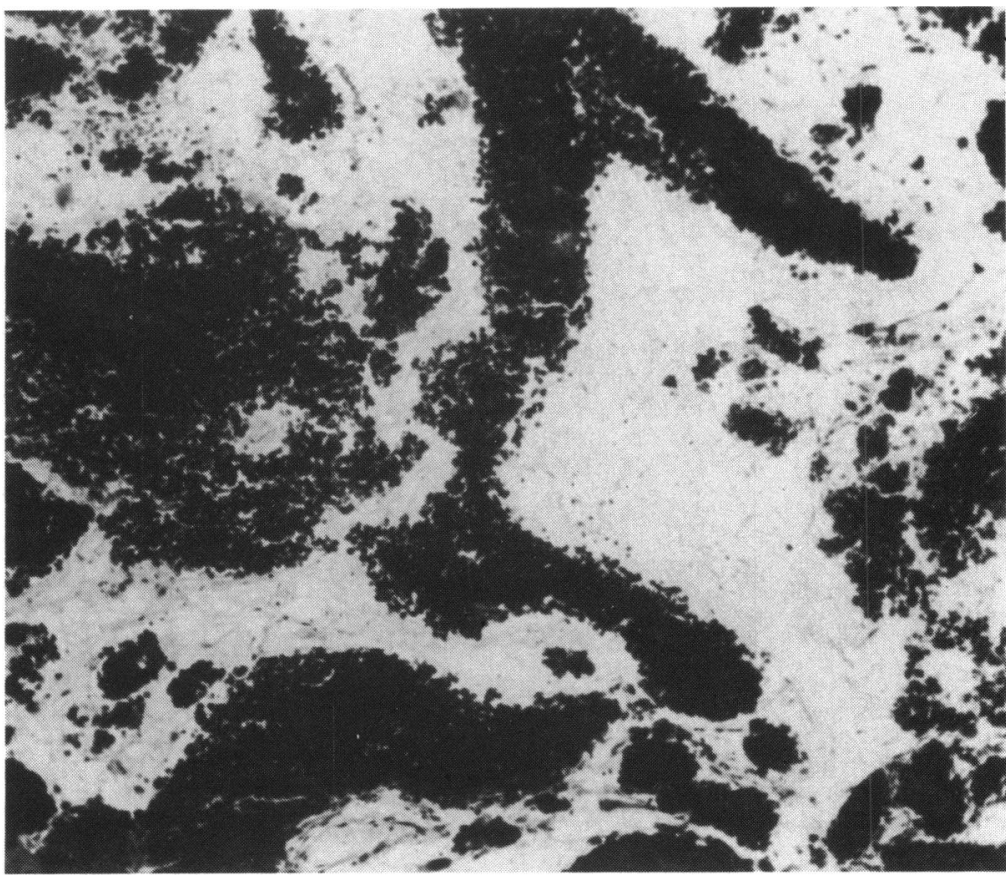

Fig. 3a Histopathological examination of the tumour shows marked calcification and tumour necrosis (Haematoxylin and eosin, $\times 30$ ).

of the 11 metaphases analysed one of the copies of chromosome 10 was missing. In addition all the cells contained structural alterations resulting in a number of marker chromosomes. A variable number of these markers were present in different metaphases. All metaphases had a marker chromosome $\left(\mathrm{M}_{1}\right)$ which is produced as a result of breakage at the secondary constriction of chromosome $1(1 \mathrm{q}-)$. $\mathrm{M}_{2}$ was present in eight of 11 metaphases. This marker is derived from chromosome 2 by addition of chromosome

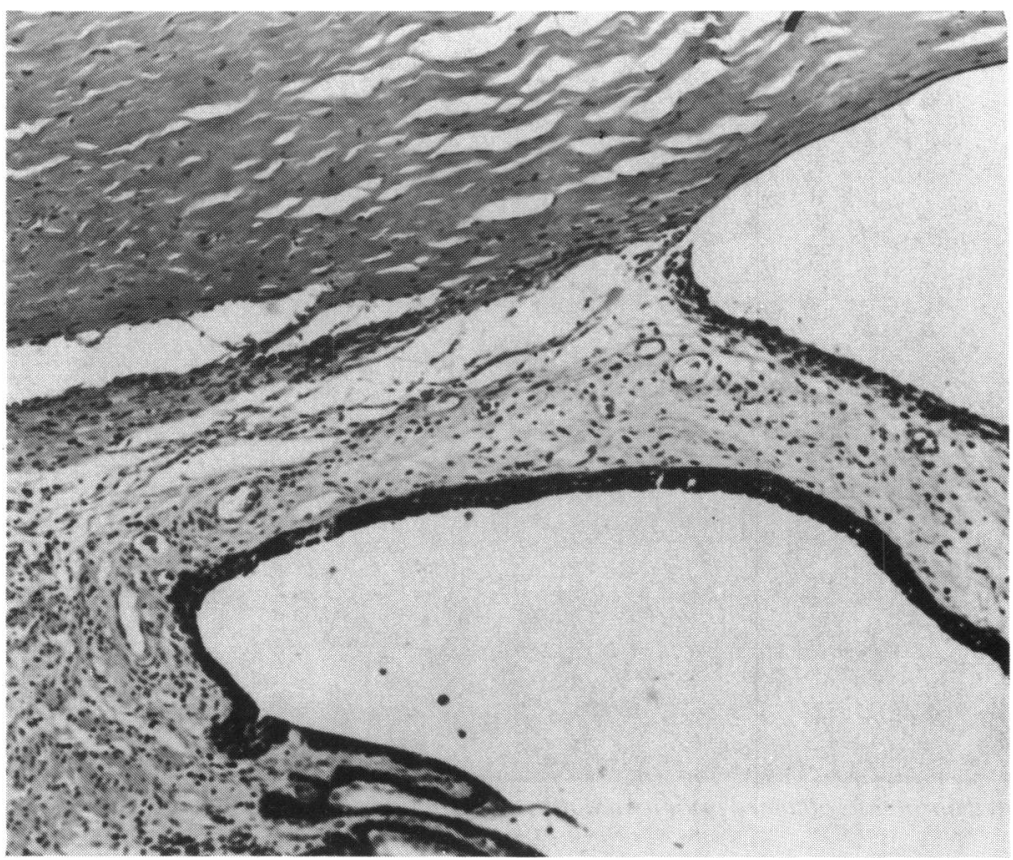

Fig. 3b Alow power photomicrograph of the enucleated eye shows marked iris neovascularisation and closure of the filtration angle. (Haematoxylin and eosin, $\times 30$ ). 
Fig. 3c Photomicrograph shows retinal changes, including preretinal fibrovascular membranes and extensive ganglion cell loss. (Haematoxylin and eosin, $\times 50$ ).

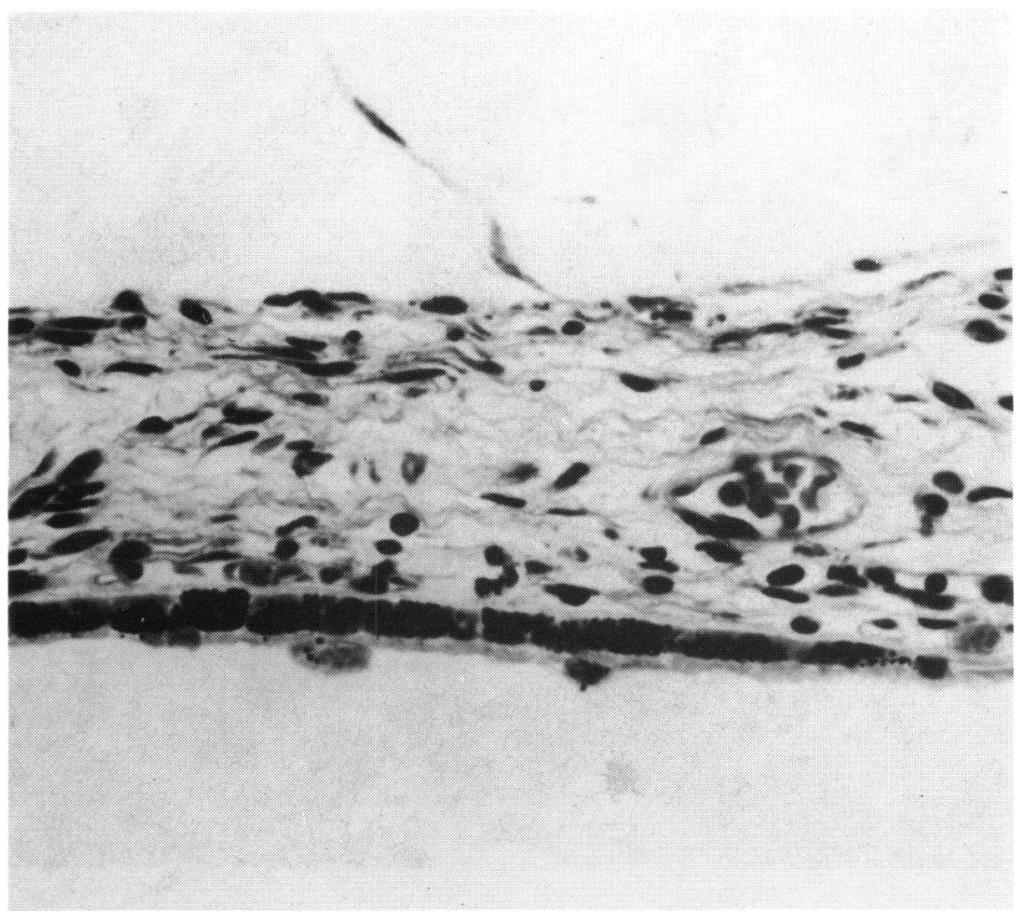

material in the distal half of chromosome $21 . \mathrm{M}_{3}$ is derived from part of chromosome 7 to which additional material was added. $\mathbf{M}_{\mathbf{4}}$ probably represents virtually the entire chromosome 8 to which additional

material is added at the end of the long arm. $\mathbf{M}_{5}$ is chromosome 9 with the major portion of the short arm deleted $(9 p-)$ and was observed in eight of 11 metaphases. $\mathbf{M}_{6}$ is produced as a translocation be-

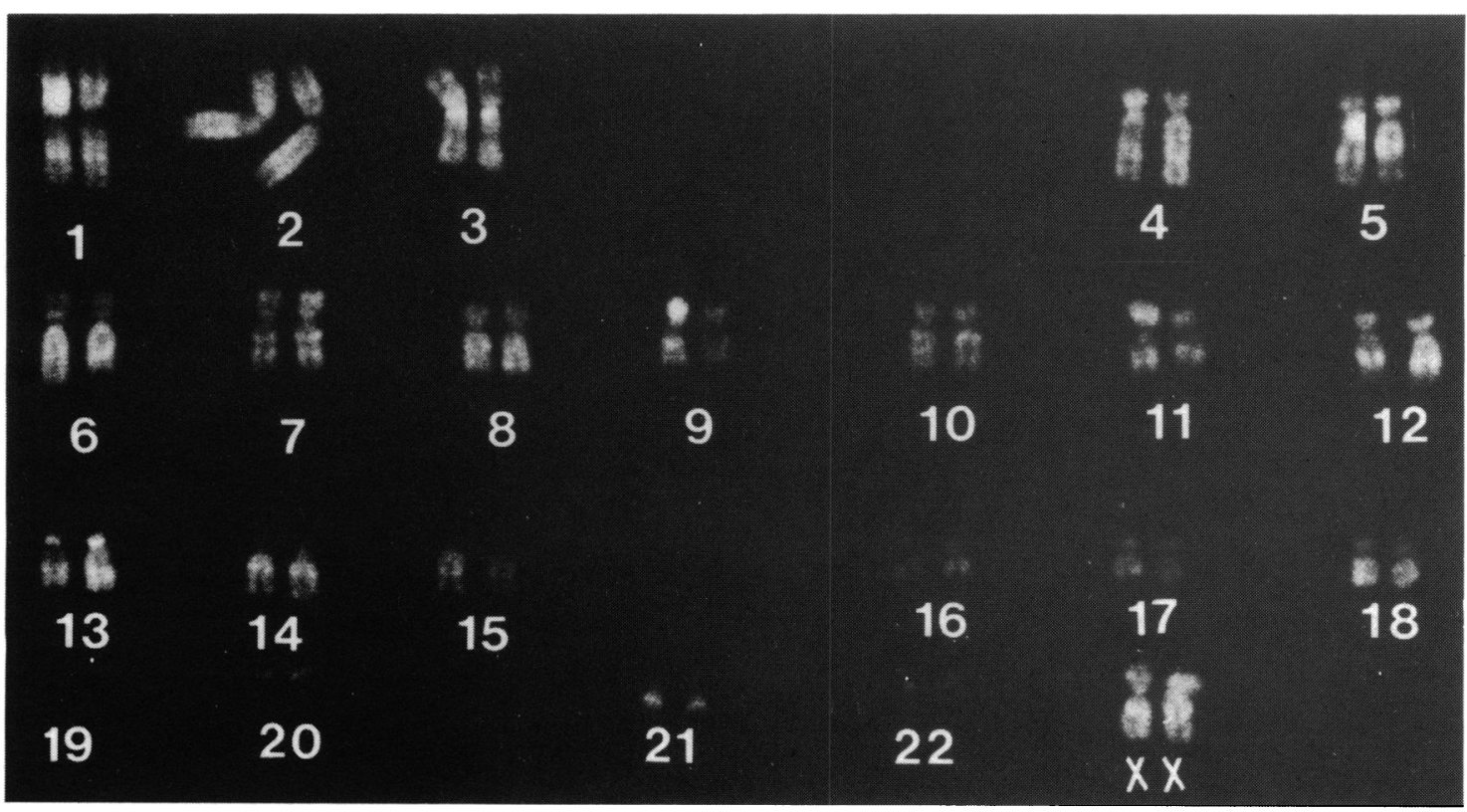

Fig. 4 A Q-banded karyotype of cultured fibroblasts obtained prior to radiotherapy. This is a normal human diploid female karyotype. 


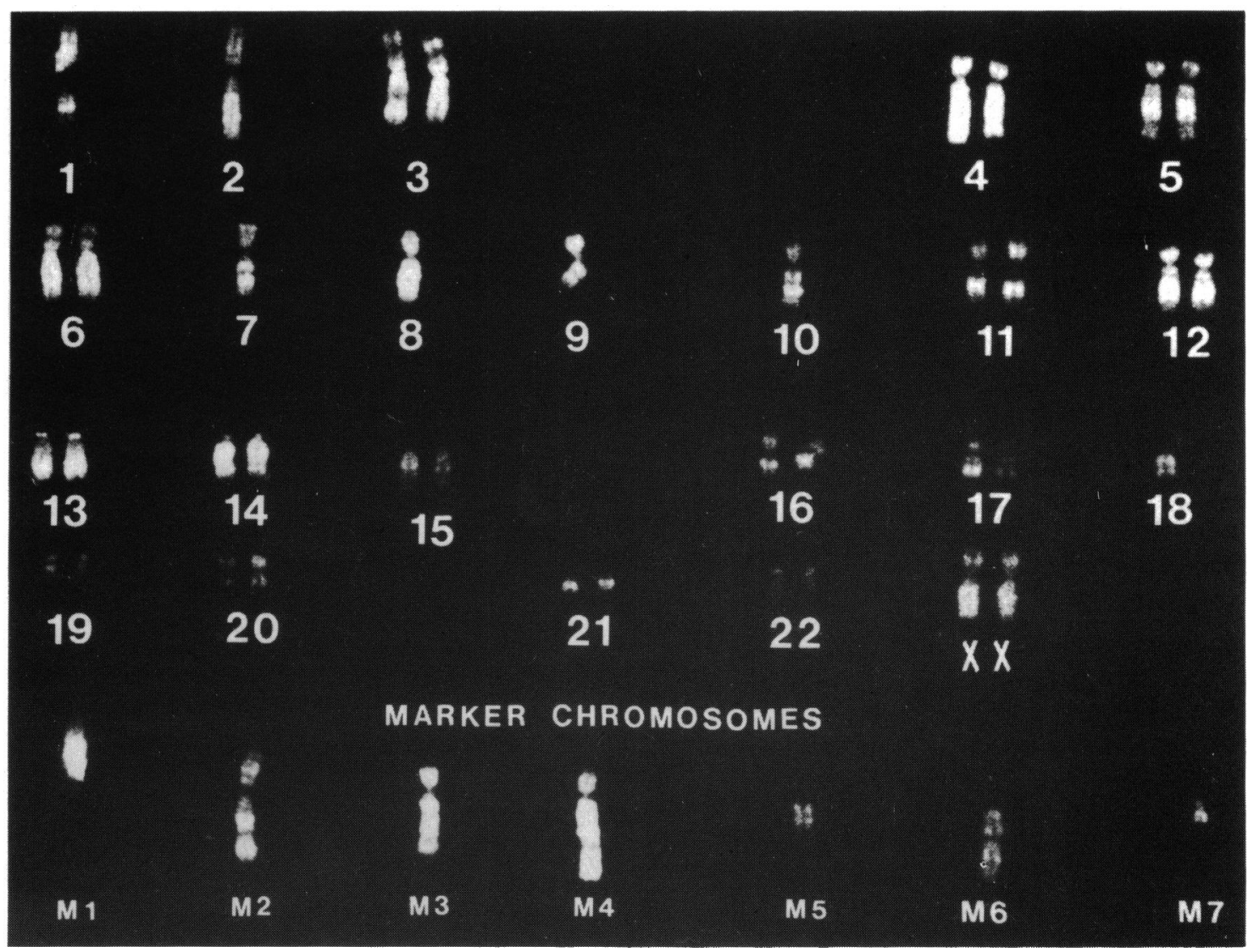

Fig. 5 A Q-banded karyotype of cultured fibroblasts following radiotherapy showing chromosome changes. This karyotype shows loss of one of each of chromosomes $1,2,7,8,9,10,18$, and presence of marker chromosomes described in text.

tween chromosome 18 and the distal half of the long arm chromosome $1 . \mathrm{M}_{7}$ is a small fragment. $\mathrm{M}_{6}$ and $M_{7}$ were present in seven of the 11 metaphases. Fig. 5 shows a Q-banded based karyotype from B-814. Both markers $\mathrm{M}_{3}$ and $\mathrm{M}_{4}$ were present in seven of 11 metaphases.

\section{Discussion}

The clinical course and pathological findings in this case represent an unusually severe ocular and orbital response to radiation therapy. Rapidly progressive iris neovascularisation with associated angle closure glaucoma and preretinal fibrovascular proliferation occurred five months after bilateral irradiation for hereditary retinoblastoma. Subsequent histopathological examination revealed that the tumours were necrotic and conformed to the clinical and histological features of radiation and subsequent regression. Although neovascularisation is frequently observed in the eyes with untreated retinoblastoma and may be associated with retinal damage, its occur- rence after radiation treatment in the doses given for this patient is unexpected ${ }^{6-8}$ In this child it followed a fulminant course involving both iris and retina. Iris neovascularisation is a known ocular tissue response following irradiation, and there is a variety of clinical and experimental evidence that ionising radiation can cause retinal vascular occlusion, neovascularisation, and preretinal proliferative changes. ${ }^{9-13}$

This study is part of an ongoing investigation of DNA repair defects in patients with hereditary retinoblastoma. We have systematically obtained skin or conjunctival biopsies from these patients and performed $x$-ray survival studies, since a defect in DNA repair may be manifested by a decrease in survival to cytotoxic agents. The pretreatment $x$-ray survival curve parameters seen in fibroblasts from this patient are consistent with the moderate radiosensitivity noted in patients with hereditary retinoblastoma. The extreme radiosensitivity in the posttreatment fibroblasts is greater than that seen in any of our study patients with hereditary retinoblastoma and is near the range of radiosensitivity seen in 
patients with ataxia telangiectasia. ${ }^{1}$ Ataxia telangiectasia is an autosomal recessive disease characterised by a striking deficiency in the repair of $x$ irradiation induced DNA damage.

In spite of the fact that the pretreatment radiosensitivity $\left(D_{0}=111\right)$ is consistent with the mean $D_{0}$ seen in fibroblasts from patients with hereditary retinoblastoma, this finding would not have predicted the extreme clinical reaction seen in this patient. The post-treatment radiosensitivity $\left(D_{0}=70\right)$ is consistent with the severe clinical radiation reaction seen in patients with ataxia telangiectasia. Since the second fibroblast biopsy was obtained from an irradiated site, we may speculate that radiation damage may have been accumulated by cells exposed to the first dose which was expressed by the postirradiation survival curve. This accumulation of $x$-ray damage may have accounted for the severe clinical reaction and the fact that extreme radiosensitivity was not seen in the preradiation experiments. This finding is consistent with our hypothesis that some patients with hereditary retinoblastoma may have a defect in the repair or accumulation of $x$-irradiation induced DNA damage which may reflect an underlying hypermutability and predispose to a second neoplasm.

When mammalian cells in culture are irradiated and a surviving clone selected from among the survivors, it has the same radiological characteristics as before radiation. Thus this case represents an unusual phenomenon of acquired radiosensitivity. ${ }^{14}$

The chromosome aberrations seen in this patient after radiotherapy are consistent with the effects of this agent on cells in vivo and in vitro. It is not known how these chromosomal aberrations relate to the development of the increased in-vitro radiosensitivity seen here. A slight increase in radiationinduced chromosome aberrations has been reported in cells from patients with hereditary retinoblastomas. It is disquieting that these severe chromoso- mal aberrations exist after radiotherapy for a patient predisposed to radiation-induced malignancy.

Supported in part by grant EY01917 from the National Eye Institute.

\section{References}

1 Weichselbaum RR, Nove J, Little JB. Skin fibroblasts from Ddeletion type retinoblastoma patients are abnormally radiosensitive. Nature 1977; 226: 726-7.

2 Weichselbaum RR, Nove J, Little JB. $X$-ray sensitivity of diploid fibroblasts from patients with hereditary and sporadic retinoblastoma. Proc Natl Acad Sci USA 1978; 75: 3962-4.

3 Sagerman RH, Cassady JR, Treeter P, Ellsworth RM. Radiation induced neoplasia following external beam therapy for children with retinoblastoma. AJR 1969; 105: 529-35.

4 Weichselbaum RR, Nove J, Little JB. $X$-ray sensitivity of fiftythree human diploid fibroblast cell strains from patients with characterized genetic disorders. Cancer Res 1980; 40: 920-5.

5 Miller DA, Firschein IL, Dev VG, Trantravahi R, Miller OJ. The gorilla karyotype. Chromosome lengths and polymorphisms. Cytogenet Cell Genet 1974; 13: 536-50.

6 Walton DS, Grant WM. Retinoblastoma and iris neovascularization. Am J Ophthalmol 1968; 65: 598-9.

7 Cheng-Minoda K. Retinoblastoma and iris neovascularization. Jpn J Ophthalmol 1971; 15: 215-37.

8 Spaulding G. Rubeosis irides in retinoblastoma and pseudoglioma. Trans Am Ophthalmol Soc 1978; 76: 584-609.

9 Winter FC, Reinhardt PR, Madden J. Ocular effects of high intensity $x$-radiation in the cat. Am J Ophthalmol 1958; 46: 114-22.

10 Howard GM. Ocular effects of radiation and photocoagulation. Arch Ophthalmol 1966; 76: 7-10.

11 Merriam GR Jr, Szechter A, Focht EF. The effects of ionizing radiations on the eye. In: Vaeth JM, ed. Frontiers of radiation therapy and oncology. Baltimore: University Park Press, 1972: 346-85.

12 Egbert PR, Donaldson SS, Moazed K, Rosenthal AR. Visual results and ocular complications following radiotherapy for retinoblastoma. Arch Ophthalmol 1978; 96: 1826-30.

13 Shukovsky L, Fletcher GH. Retinal and optic nerve complications in a high dose irradiation technique of ethmoid sinus and nasal cavity. Radiology 1972; 104: 629-34.

14 Little JB, Williams JR. Affects of ionizing radiation on mammalian cells. Handbook of physiology. Bethesda: American Physiologic Society, D N K Lee, 1977: 127-56.

Accepted for publication 12 September 1985. 